\title{
Detection of Circulating Tumor Cells and Its Application in Prostate Cancer
}

\author{
Jin-Qi Song, Ya-Nan Zhou, Gang-Liang Tu, Chang-Li Xu, Hui Xu \\ Department of Urology, the Affiliated Hospital of Chengde Medical College, Chengde, PR China.
}

\begin{abstract}
In recent years, circulating tumor cells have become the focus of tumor research. In-depth study of the role of circulating tumor cells in the genesis, development, and evolution of tumors will be of great significance for the early detection, early diagnosis, early treatment, and prognosis of tumors. Prostate cancer is one of the common male malignant tumors, and the role of circulating tumor cells in prostate cancer has been increasing year by year. This article will focus on the progress of circulating tumor cells detection and its application in prostate cancer.
\end{abstract}

Keywords: Circulating Tumor Cells, Prostate Cancer.

\section{Introduction}

In recent years, prostate cancer $(\mathrm{PCa})$ has been on the rise in morbidity and mortality among middle-aged and elderly men in China, placing a huge burden on the national medical system. Studies have shown that PCa is one of the most common malignant tumors in males in western countries. In 2015, about 27,540 patients died of $\mathrm{PCa}$ in the United States which is the second common cause of death after lung cancer. ${ }^{1} \mathrm{PCa}$ is one of the most common male malignant tumors, hence early screening plays an important role in detecting the disease in an early stage. Due to the lack of specificity of clinical symptoms, patients often come to the hospital in the late stage; relevant data showed that the five-year survival rate of early localized PCa is close to $100 \%$, while the survival rate of advanced metastatic cancer significantly decreases as low as to $33.5 \% .^{2}$ Prostate-specific antigen (PSA) is a tumor marker for routine diagnosis of PCa, but it has its limitations. Core needle biopsy of the prostate is invasive, with risks of bleeding and infection. Circulating tumor cells (CTC) detection has been applied to monitor the metastasis of $\mathrm{PCa}$, and guide the tumor diagnosis and plan the treatment. It has certain relevance with pathologic stage of $\mathrm{PCa}$, however, it is reported with mixed results. ${ }^{3}$ This review will focus on the common CTC detection methods and their application in PCa in recent years.

\section{Definition and Main Characteristics of} Circulating Tumor Cells

According to previous studies, the concept of CTC was proposed in 1869 when tumor-like cells were found in the peripheral blood of a patient who died of cancer at autopsy. CTC from patients with tumors, primary tumors, most fall off after death, only a handful of highly dynamic cells with metastatic potential after the escape from the body's immune surveillance run in the circulation of the blood system. These cells get attached to form tiny tumor emboli for their survival. Under certain favorable conditions in a distant site, they clump as the formation of metastases. The detection of CTC in the blood circulation does not mean that metastasis has occurred, but the possibility of metastasis is substantially increased. Therefore, tumor cells from the primary tumor

\section{Correspondence}

Hui Xu, Department of Urology, the Affiliated Hospital of Chengde Medical College, Chengde, Hebei, China, chengdexuhui@163.com. 
and metastatic tumor that have been actively or passively injected into the blood and survived immune killing are called CTC. ${ }^{4}$ CTC in the blood from the primary and metastatic foci can grow back to the primary site of the tumor and grow in tissues and organs other than the primary site to form new tumors, which is the selfplanting or cross-planting of the tumor. ${ }^{5}$

At present, it is believed that the characteristic markers of CTC are: $\mathrm{N}$-Calcium mucin (membrane protein of calcium mucin family), vimentin (a structural cytoskeletal protein), the nuclear localization of $\beta$-catenin protein and transcription factors such as SNAIL, SNAI2, TWIST, homeobox1-ZEB1, ZEB2, and TCF3. ${ }^{6}$ CTC can be isolated by identifying epithelial markers on the cell surface. However, it has been reported that the epithelial-mesenchymal transformation mechanism can cause epithelial cells to lose polarity, resulting in loose intercellular connections and eventually become cells with the morphology and characteristics of mesenchymal cells. This increases the difficulty of obtaining CTC. ${ }^{7}$

\section{Circulating Tumor Cells Enrichment Method}

CTC entering the blood circulation will stimulate the immune response of the body, most CTC will be eliminated by the immune system of the body, and thus the final numbers of CTC remaining in the peripheral blood circulation is very small. In patients with metastatic cancer, there is only about one CTC in every ${ }^{105-107}$ peripheral blood monocytes. Therefore, before CTC detection and analysis, a large number of mononuclear cells need to be isolated before CTC enrichment. The advantages and disadvantages of the enrichment results will directly affect the subsequent detection, which requires a high degree of CTC enrichment method. ${ }^{8}$

The enrichment technique of CTC is mainly based on morphological or immunological characteristics. At present, there are two main enrichment methods: ${ }^{1}$ Using the physical properties of cells, such as size, shape, density, and charge, etc. (a) Size and shape: tumor cells are larger than peripheral blood cells, the use of membrane filtration method. CTC cannot be separated from normal cells through a filter membrane of $5-10 \mu \mathrm{m}$ aperture. However, due to small diameter, a small number of tumor cells can pass through the pore diameter of the filter membrane, resulting in low sensitivity for enrichment. ${ }^{9}$ The microfluidic technology operates the liquid in the micron channel, which has the advantages of simplicity, rapidity, low cost and high flux. The new microfluidic chip can quickly and accurately capture and count CTC from whole blood samples, and the screened CTC can maintain the activity, which is conducive to subsequent analysis and research. ${ }^{8}$ (b) Density gradient separation method: the use of the density difference between the cells, the use of stratified fluid to separate the tumor cells from many cells. Oncoquick, with its built-in porous barrier, blocks CTC and allows red blood cells and some white blood cells to pass through, greatly increasing enrichment efficiency. However, studies have shown that although the density gradient separation method is simple and low-cost, its sensitivity and specificity are not very high, which may easily lead to the loss of some tumor cells. Therefore, this method cannot be popularized. ${ }^{10}$ (c) Two-dimensional electrophoresis: due to the phenotype, physiological state, and morphology of different cells, resulting in different dielectric properties, which can be divided into the basis of tumor cells. Twodimensional electrophoresis does not need to rely on cell surface markers, which may separate CTC with charge, and the CTC isolated is active, facilitating further research. ${ }^{11}$ (2)Use of cell biochemistry: this is based on a specific antigen-antibody reaction principle, in which the immunomagnetic separation method is most commonly used. The immunomagnetic particles coated with specific antibodies bind to specific antigens on the cell surface, and the magnetic field is used to separate the cells that have specific reactions. Immunomagnetic bead separation techniques include positive sorting and negative enrichment. Positive sorting is the combination of specific antibodies and specific antigens such as epithelial adhesion molecules on the surface of tumor cells to separate CTC. ${ }^{12}$ Negative enrichment is the removal of white blood cells from the blood by combining specific antibody with the common antigen CD45 on the surface of white blood cells to obtain the enriched CTC. Therefore, the negative enrichment method is still applicable when the surface antigen of tumor cells cannot be fully expressed. ${ }^{13}$

\section{Application Progress of Circulating Tumor Cells Detection Technology in PCa}

The gold standard for diagnosing PCa is a classic biopsy, but this does not detect changes in the primary tumor. The occurrence of PCa has tissue heterogeneity and time heterogeneity i.e. it is difficult for biopsy to detect 
the tissue changes of uncollected lesions and the lesions after metastasis. CTC can overcome the defects in tissue biopsy (tissue heterogeneity and time heterogeneity), to improve the efficiency of biopsy.

The Cell Search system is currently the only FDA approved test for predicting the prognosis of prostate cancer. It is a semi-automated technique that uses cells derived from the epithelium of immune magnetic nanoparticles to work. The particle contains a nanomagnetic core in which immune nanomagnetic particles coated with anti-specific antibodies bind to cells in the blood that express epithelial cell adhesion molecules (specific antigens). Then, in a magnetic field, the Cell Search system uses cytokeratin antibody, nuclear fluorescent dye and CD45 antibody combined with fluorescent dye to jointly recognize CTC, and defines cells of epithelial cell adhesion molecule $(+)$, cytokeratin (+), DAPI (+) and CD45 (-) as CTC. ${ }^{7}$ Due to its high degree of automation and low human influence, Cell Search system has high specificity and repeatability and can enrich and count CTC. ${ }^{8}$ The system has been widely used in prostate cancer research. Studies have reported that among 188 peripheral blood samples collected from 123 patients with metastatic castration-resistant prostate cancer, $57 \%$ of the samples detected $\geq 2$ CTC per $7.5 \mathrm{ml}$ of peripheral blood, and about $14 \%$ of the samples detected $\geq 50$ CTC. ${ }^{14}$ Recent studies have also reported using the Cell Search system in clinical trials to evaluate the efficacy of combined docetaxel and atracentam chemotherapy, suggesting that baseline CTC counts are independent risk factors for castration-resistant prostate cancer. ${ }^{15}$ However, when tumor cells undergo an epithelial-mesenchymal transformation mechanism, the expression of epithelial adhesion molecules changes, which will reduce the sensitivity of this system to detect CTC. However, some studies have reported the expression of epithelial-mesenchymal transformation mechanism phenotypic markers (such as CD146) in the CTC of epithelial cell adhesion molecules (-), which is expected to improve the sensitivity of this system. ${ }^{16}$

The Adna test system is similar to the Cell Search system, it combines the immunomagnetic bead separation technology with the gene reverse transcription technology. It combines immunomagnetic beads with epithelial cell adhesion molecules on the surface of CTC and then conducts directional movement under the effect of the external magnetic field to screen and isolate target cells. After the target cells dissolve, RNA is extracted; the transcriptional changes of the cells are further analyzed by RT-PCR, and CTC is detected. RTPCR is mainly used to detect CTC by amplification of mRNA, which is the marker of tumor cells. The standard Adna Test system includes primers for PSA, prostatespecific membrane antigen, and epidermal growth factor receptor (EGFR), defining the cells that detect these genes as CTC. It had been reported that CTC was detected in 11 out of 16 castration-resistant prostate cancer patients without chemotherapy by Adna Test system. ${ }^{17}$ The Cell Search system and the Adna Test system share a common shortcoming: the cell surface must express epithelial cell adhesion molecules, and tumor cells with no or low expression are excluded. This defect limits the identification of CTC independent of epithelial adhesion molecules. High-throughput imaging can compensate for this by detecting a very small number of fluorescein-labeled CTC in tens of thousands of blood cells. At present, the Epic platform of us Epic Sciences and AccuCyte CyteFinder system of us RareCyte can use this technology for CTC detection. The epic platform uses fiber array scanning technology and advanced image acquisition technology to detect CTC. It can detect conventional CTC, small CTC, circulating tumor cell cluster or circulating tumor microemboli (CTM), apoptotic CTC and CTC without expression of cytokeratin. AccuCyte CyteFinder system used AccuCyte kit to isolate nucleated cells in the blood through different densities, and then conducted staining and immunofluorescence analysis. Finally, high-throughput imaging technology and a CyteFinder scanning microscope were used to identify suspected CTC. ${ }^{13}$ In previous studies, CTC of 17 patients with metastatic prostate cancer was determined by AccuCyte CyteFinder, and the average number of CTC calculated was more than $34 \%$ higher than that of the Cell Search system. ${ }^{18}$

Flow cytometry is a technique for the detection and analysis of cellular and subcellular structures. It can quickly measure the biophysical, biochemical, and other characteristic parameters of cells, and can separate the specified cell subsets according to the preselected parameter range. It has been outlined that in vivo fluorescent flow cytometry can monitor CTC in blood non-invasively and in real-time. If the combination of 
photo-acoustic flow cytometry and fluorescent flow cytometry can complement defects, it can be better applied to the detection of CTC. ${ }^{19}$ The sensitivity of RTPCR was higher than that of flow cytometry. However, false-positive results are prone to occur, and CTC counting and detection of CTC morphology cannot be performed by this technique. ${ }^{20}$ RT-PCR is based on a PCR method, thus, the amount of DNA can be measured to identify nucleic acids from cells responsible for the transfer process. As it can only detect mRNA from living cells, it cannot be used to distinguish DNA-derived apoptotic cells from living cells. RT-PCR is more helpful in characterizing CTC than in detecting them. ${ }^{21,22}$

In a study done by Ali et. al, among 9 patients with small-volume PCa after radical prostatectomy, CTC was detected in 7 patients and PSA level was detected in 7 patients, while serum PSA was detected in only 2 of them.23 Likewise in another study, the detection rates of CTC $\geq 2$ in patients with gastric cancer, lung cancer, esophageal cancer, and breast cancer were $86.21 \%$, $71.33 \%, 78.35 \%$, and $76.77 \%$, respectively. ${ }^{24}$ Among 161 PCa patients studied by Miao YX et. al, the CTC detection rate was $74.53 \%$, among which the CTC detection rate of early PCa ( $\leq$ pT3a stage) was $65.22 \%$, far higher than that of the control group (7.14\%) and benign prostate disease (20\%). CTC $\geq 2$ was used as the bound, the sensitivity was $74.53 \%$, and the specificity was $87.01 \%$. This indicates that CTC detection is helpful for the early diagnosis and adjuvant therapy of $\mathrm{PCa} .{ }^{3}$ At the same time, this study found that there was a significant correlation between CTC and PCa Gleason score and pathological grade. With the increase of Gleason score and pathological grade, the number of CTC increased significantly, especially the proportion of CTC count $\geq 5$. Olmos et. al, studied 119 patients with metastatic castrationresistant $\mathrm{PCa}$, and the results indicated that CTC count $\geq 5$ detected at any time was an independent and strong prognostic predictor, indicating poor prognosis and short overall survival. ${ }^{25}$ Davis et. al, conducted the study in $97 \mathrm{PCa}$ patients undergoing radical prostatectomy as case and 25 men with an increased prostate-specific antigen and no tumor detected on extended prostate biopsy as the control group to analyze circulating tumor cells using the CellSearch System, the results showed that there was no correlation of Circulating tumor cell with tumor volume, pathological stage or Gleason score. ${ }^{26}$ Due to the inert characteristics of the tumor, the longer the clinical incubation period of PCa patients, the lesser the increase in the number of CTC in peripheral blood; and the tumor invasion is relatively low. Contrastingly, in a study done by Oscar B Goodman et. al, the CTC number in peripheral blood was significantly increased in patients $<60$ years old with high Gleason score and short incubation period, indicating higher tumor invasion. Moreover, a large number of clinical trials still need to be done to verify whether CTC count can be used alone or in combination with some prognostic factors for the diagnosis and prognosis of $\mathrm{PCa} .{ }^{27}$

\section{Conclusion}

With the continuous innovation of CTC enrichment and detection technology. To make up their shortcomings and improve the sensitivity and specificity of detection, multiple methods of joint detection of CTC will be a future development trend. Although CTC can be isolated and counted by many techniques, testing at the molecular and genetic levels is currently very challenging. It is believed that with the in-depth study of liquid biopsy technology, soon, this technology will be widely used in the early screening, early diagnosis, clinical staging, pathological grading, treatment guidance, prognosis assessment, realtime monitoring of tumor recurrence and other aspects of PCa, bringing great benefits to cancer patients.

\section{References}

1. Pantel K, Brakenhoff RH, Brandt B. Detection, clinical relevance, and specific biological properties of disseminating tumor cells. Nat Rev Cancer. 2008; 8(5):329-340.

2. Zhao J, Li XL, Suo ZH. Research progress of circulating tumor cells and free DNA methylation in prostate cancer. Cancer Progress. 2016; 14(4): 308310,315 .

3. Miu YX, Jiang WR, Guo MQ, et al. Clinical application of circulating tumor cells in the diagnosis of prostate cancer. Chin J lab Med. 2019; 42(3): 198-203.

4. De Mattos-Arruda L, Cortes J, Santarpia L, et al. Circulating tumor cells and cell-free DNA as tools for managing breast cancer. Natrev Clin Oncol. 2013; 10(7): 377-389.

5. Kim MY, Oskarsson T, Acharyya S, et al. Tumor selfseeding by circulating cancer cells. Cell. 2009; 139(7): 1315-1326.

6. Stelcer E, Konkol M, Gleboka A, et al. Liquid biopsy 
in oligometastatic prostate cancer-a biologist's point of view. Front Oncol. 2019; 9(1):775.

7. Tang Y. Correlation between peripheral circulating tumor cells and clinical characteristics in elderly patients with prostate cancer. Soochow University. 2017; 13(1): 1-45.

8. Xie JH, Pan HX, Li WD. Detection and clinical application of circulating tumor cells in prostate cancer. J Clin Urology. 2016; 31(7): 674-678.

9. Coumans Frank AW, Van DG, Beck M, et al. Filtration parameters influencing circulating tumor cell enrichment from whole blood. Plos One. 2013; 8(4): 1-9.

10. Hu YQ, Xie SG, Zheng Y, et al. Review on enrichment and detection of circulating tumor cells. Cancer Res Prev Treat. 2014; 41(7): 834-838.

11. Peter RC Gascoyne, Sangjo S. Isolation of circulating tumor cells by dielectrophoresis. Cancers. 2014; 6(10): 545-579.

12. Cohen SJ, Alpaugh RK, Gross S, et al. Isolation and characterization of circulating tumor cells in patients with metastatic colorectal cancer. Clin Colorectal Canc. 2006; 6(2): 125-132.

13. Powell Ashley A. Talasaz AA, Zhang HY, et al. Singlecell profiling of circulating tumor cells: transcriptional heterogeneity and diversity from breast cancer cell lines. Plos One. 2012; 7(5): 1-11.

14. Shi Y, Peng Z, Liu KY, et al. Application progress of new detection techniques for peripheral blood circulating tumor cells in prostate cancer. Cancer Research and Clinic. 2018; 30(11): 789-792.

15. Amir G, Benjamin E, David IQ, et al. Circulating tumor cell counts are prognostic of overall survival in SWOG S0421: a phase III trial of docetaxel with or without atrasentan for metastatic castration-resistant prostate cancer. J Clin Oncol. 2014; 32(11): 11361142 .

16. Bianca M, Jaco K, Joan BV, et al. Detection of circulating tumor cells in breast cancer may improve through enrichment with anti-CD146. Breast Cancer Res Tr. 2011; 127(1):33-41.

17. Todenhöfer T, Hennenlotter J, Feyerabend S, et al. Preliminary experience on the use of the adna test system for detection of circulating tumor cells in prostate cancer patients. Anticancer Res. 2012; 32(8): 3507-3513.
18. Stilwell JL, Drovetto N, Ramirez AB, et al. Clinical performance of the AccuCyte-CyteFin-der System, a dual-technology platform for comprehensive collection and high-resolution imaging of circulating tumor cells. Cancer Res. 2015; 75(15): 723-748.

19. Dmitry AN, Mustafa S, Ekaterina IG, et al.Synergy of photoacoustic and fluorescence flow cytometry of circulating cells with negative and positive contrasts. J Biophotonics. 2013; 6(5): 425-434.

20. Wang ZD, Zhao WH, Li S. Advance in the detection of circulating tumor cells. Chin J Cancer Prev Treat. 2014; 21(17): 1391-1394.

21. Broersen LH, Van Pelt GW, Tollenaar RA, et al. Clinical application of circulating tumor cells in breast cancer. Cell Oncol. 2014; 37(1): 9-15.

22. Andergassen U, Kölbl AC, Mahner S, et al. Realtime RT-PCR systems for CTC detection from blood samples of breast cancer and gynecological tumor patients. Oncol Rep. 2016; 35(4): 1905-1915.

23. Amina A, Bungo F, Paul OP, et al. Assessment of circulating tumor cells (CTCs) in prostate cancer patients with low-volume tumors. Pathol Int. 2010; 60(10): 667-672.

24. Li Y, Ma G, Zhao P, et al. Improvement of sensitive and specific detection of circulating tumor cells using negative enrichment and immunostaining-FISH. Clin Chim Acta. 2018; 485(1): 95-102.

25. Olmos D, Arkenau HT, Ang JE, et al. Circulating tumor cell (CTC) counts as intermediate endpoints in castration-resistant prostate cancer (CRPC): a single-center experience. Ann Oncol. 2009; 20(1): 27-33.

26. Davis JW, Nakanishi H, Kumar VS, et al. Circulating tumor cells in peripheral blood samples from patients with increased serum prostate-specific antigen: initial results in early prostate cancer. J Urol. 2008; 179(6): 2187-2191.

27. Oscar BG, Louis MF, James TS, et al. Circulating tumor cells in patients with castration-resistant prostate cancer baseline values and correlation with prognostic factors. Cancer Epidem Biomar. 2009; 18(6): 1904-1913.

28. Lin ZQ, Wang SB, Liang $Y$, et al. Progress and application of liquid biopsy in prostatic cancer. J Clin Urology. 2019; 34(2):154-156. 